\title{
Dielectric and Microwave Properties of Graphene Nanoplatelets /Carbon Black Filled Natural Rubber Composites
}

\author{
Omar A. Al-Hartomy ${ }^{1,2}$, Ahmed A. Al-Ghamdi ${ }^{1}$, Falleh Al-Salamy ${ }^{3}$, Nikolay Dis hovsky ${ }^{4, *}$, \\ Rossitsa Shtarkova ${ }^{5}$, Vladimir Ilie $v^{6}$, Farid El-Tantawy ${ }^{7}$ \\ ${ }^{1}$ Department of Physics, Faculty of Science, King Abdulaziz University, Jeddah, 21589, Saudi Arabia \\ ${ }^{2}$ Department of Phy sics, Faculty of Science, University of Tabuk, Tabuk, 71491, Saudi Arabia \\ ${ }^{3}$ Department of Mathematics, Faculty of Science, King Abdulaziz University, Jeddah, 21589, Saudi Arabia \\ ${ }^{4}$ Department of Polymer Engineering, University of Chemical Technology and Metallurgy, 8 Kl. Ohridski blvd., 1756, Sofia, Bulgaria \\ ${ }^{5}$ Department of Chemistry, Technical University, 8 Kl. Ohridski blvd., 1000, Sofia, Bulgaria \\ ${ }^{6}$ College of Telecommunications and Posts, Sofia, Bulgaria \\ ${ }^{7}$ Department of Phy sics, Faculty of Science, Suez Canal University, Ismailia, Egypt
}

\begin{abstract}
Natural rubber (NR) based nanocomposites containing a constant amount (50 phr) of standard furnace carbon black and graphene nanoplatelets (GNP) in concentrations from 1 to $5 \mathrm{phr}$ have been prepared. Their dielectric (dielectric permittivity, dielectric loss) and mic rowave properties (coefficients of absorption and reflection of the electro magnetic waves and electromagnetic interference shielding effectiveness) have been investigated in the $1-12 \mathrm{GHz}$ frequency range. The results achieved allow us to reco mmend GNP as second filler for natural rubber based composites to afford specific absorbing properties.
\end{abstract}

Keywords Natural Rubber Composites, Graphene Nanoplatelets, Dielectric and Micro wave Properties

\section{Introduction}

Significant scientific and technological interest has focused on carbon - polymer nanocomposites over the last two decades. The specific development of poly meric nanocomposites based on conventional poly mers and conductive carbonaceous materials has drawn much attention as a route to obtain new materials with new structural and functional properties superior to those of the pure components that find applications in many industrial fields. Control of the size, shape and surface chemistry of the reinforcement materials are essential in the development of materials that can be used to produce devices, sensors and actuators based on the modulation of functional properties as[1]. At present, nanocomposites employing carbon-based reinforcement materials are dominated by carbon nanotubes (CNTs) as[2-4]. However, the development of CNT-reinforced composites has been impeded by both their difficult dispersion in the polymer matrix and their high cost.

The discovery of graphene[5] and the subsequent development of graphene-based polymer nanocomposites is

* Corresponding author:

dishov@uctm.edu (Nikolay Dishovsky)

Published online at http://journal.sapub.org/ijmc

Copyright @ 2012 Scientific \& Academic Publishing. All Rights Reserved an important addition in the area of nanoscience and technology. Graphene is an allotrope of carbon, whose structure is one-atom-th ick planar sheets of $\mathrm{sp}^{2}-$ bonded carbon atoms packed in a honeycomb lattice. It is the basic structural element of some carbon allotropes including graphite, charcoal, carbon nanotubes and fullerenes [6].

Compared to carbon nanotubes, as well as its high aspect ratio and low density graphene has attracted considerable attention because of its unique and outstanding mechanical, electrical and electronic properties (e.g. exceptional in -plane electrical conductivity - up to $\sim 20,000 \mathrm{~S} / \mathrm{cm}$ and highest thermal conductivity - up to $\sim 5,300 \mathrm{~W} / \mathrm{mK}$ ).

All these unique properties in a single nanomaterial have made physicists, chemists and material scientists exited about graphene's potential. The history, che mistry, preparation methods and possible applications of graphene are reviewed in[7-9]. Another new review focused on trends and frontiers in graphene-based polymer nanocomposites was published an year ago[10]. Recently, the trustees of Princeton University received a patent for graphene-elastomer nanocomposites where functionalized graphene sheets had been dispersed in vulcanized natural rubber, styrene butadiene rubber, Ps-isoprene-Ps and Polydimethylsiloxane (PDMS)[11]. The authors concluded that graphene-rubber nanocomposites possess qualities like those of carbon nanotube composites but are much cheaper to make. 
Nanostructure material might be one of the most promising materials to enhance the EM wave absorption ability owing to its higher surface area, more surface ato ms, multireflection, and thus larger dielectric or/and magnetic loss. Combination of polymers and nanomaterials is able to integrate the large electric/magnetic loss of inorganic materials and the easy tenability of polymer and is possibly an optimal strategy to design excellent EM wave absorption materials.

Lately the research works appear about a possible applications of nanocomposites based on elastomeric matrices and carbon nanotubes as filler in microwave absorbers for deciding of electromagnetic interference (EMI) and electromagnetic compatibility (EMC) problems[12-21]. The polymer matrices used in these cases are usually epoxy resin, acrylonitrile-butadiene rubber, styrene-butadiene rubber, silicone rubber, polyurethane rubber. Comparatively it is rare to find out investigations on nanocomposites based on natural rubber (NR) and carbon nanotubes and this is in the last years [22- 25].

The data about graphene influence on the microwave properties of the elastomeric composites are very scarce. Y. Chen et al. used functionalized graphene-epoxy composites as lightweight shielding materials for electromagnetic radiation[26]. I. M. De Rosa and co-workers have a wide expertise in the design of micro/nanocomposites based on carbon fibers and carbon nanotubes, for the realization of high performing radar absorbing screens, with tailored properties [27-29]. In a recent paper[30], the authors have accomplished a Salisbury screen that consis ts of three layers. The second layer (the spacer) is a low-loss-tangent nanocomposite based on a Bisphenol-A based epoxy resin filled with g raphene nanoplatelets (GNPs) at $0,5 \%$ and $1 \mathrm{wt} \%$. The real and imaginary parts of the complex effective permittivity within the 8-18 GHz range of the nanocomposite filled with GNPs have been shown. It has been observed that the real part of the effective permittivity is nearly constant.

The price of GNPs is still significantly higher than standard furnace carbon black. In this context, the aim of this study is to determine whether the addition of small quantities (1-5 phr) GNP to one standard significantly greater amount of active furnace carbon black ( $50 \mathrm{phr}$ ) can be used as a way to modify and control, primarily to improve the dielectric (dielectric permittivity, dielectric loss) and microwave properties (coefficient of reflection, coefficient of attenuation, electro magnetic interfe rence shielding effectiveness) of composites based on natural rubber in the high frequency range 1-12 GHz. Data for such a study of those combinations of fillers that we mentioned was not found in the literature.

\section{Experimental}

\subsection{Materials}

Natural rubber (SMR 10) was purchased from North Special Rubber Corporation of Hengshui, Hebei Province, China. Other ingredients such as zinc oxide $(\mathrm{ZnO})$, stearic
$\operatorname{acid}(\mathrm{SA}), \mathrm{N}-(1,3-d$ imethylbuthyl)-N'-phenyl-p-phenylenediamine (Vulkanox 4020, produced by Lanxess), MBTS (Vulkacit DM, produced by Lan xess) and sulphur (S) were commercial grades and used without further purification.

\subsection{Characterization of the Graphene used}

Graphene produced by Hayzen Engineering Co., Ankara, Turkey was used in our investigation (Figure 1). Graphene nanoplatelets (GNP) have a "platelet" morphology, meaning they have a very thin but wide as pect ("sheet"-like structure). Aspect ratios for this material can range into the thousands. Each particle consists of several sheets of graphene with an overall thickness of $50 \mathrm{~nm}$ and average plate diameter 40 microns. The pattern taken in SAED regime shows a considerable number of spot-like reflections typical for single-crystal structures (Figure 1 - in the right angle).

\subsection{Characterization of Carbon Black used}

The specific structure features of carbon black used (Corax N 220, produced by Evonik) are shown in Figure 2.

It can be seen that the primary particle size of the carbon black used is about $20 \mathrm{~nm}$, but their capability to form secondary aggregates and agglomerates is considerable. SAED indicates the absence of crystal structures.

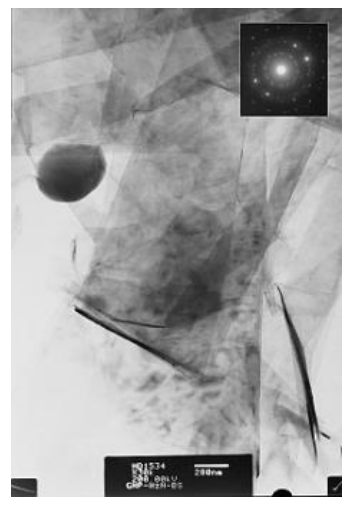

Figure 1. TEM Micrographs of GNP in transmission regime and selected area electron diffraction regime (insert in the right comer)

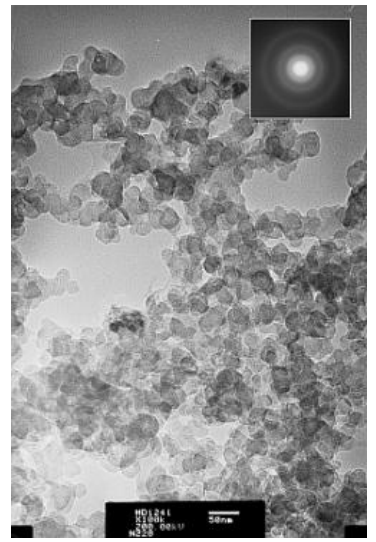

Figure 2. TEM micrographs of furnace carbon black Corax N220 particles together with SAED (insert in the right corner)

\subsection{Preparation and Vulcanization of Rubber Compounds}


Table 1 summarizes the formu lation characteristics of the rubber compounds (in phr) used for the investigations.

Table 1. Composition of the rubber compounds studied

\begin{tabular}{|c|c|c|c|c|}
\hline Ingre dients & NR 1 & NR 2 & NR 3 & NR 4 \\
\hline Natural Rubber & 100 & 100 & 100 & 100 \\
\hline Foaming agent & 8 & 8 & 8 & 8 \\
\hline Stearic acid & 1 & 1 & 1 & 1 \\
\hline ZnO & 4 & 4 & 4 & 4 \\
\hline Processing oil & 10 & 10 & 10 & 10 \\
\hline Carbon black N220 $^{2}$ & 50 & 50 & 50 & 50 \\
\hline GNP & 0 & 1 & 3 & 5 \\
\hline MBT S $^{\mathrm{a}}$ & 2 & 2 & 2 & 2 \\
\hline TMTD $^{\mathrm{b}}$ & 1 & 1 & 1 & 1 \\
\hline IPPD 4020 $^{\mathrm{c}}$ & 1 & 1 & 1 & 1 \\
\hline Sulphur $^{2}$ & 2 & 2 & 2 & 2 \\
\hline
\end{tabular}

${ }^{\text {a }}$ Mercapto benzothiazole sulphenamide

${ }^{\mathrm{b}}$ Tetramethyl thiuram disulphide

${ }^{\mathrm{c}}$ Dimethylbutyl-phenyl-p-phenylendiamine

The rubber compounds were prepared on an open two-roll laboratory mill (L/D 320x360 and friction 1.27 ) by incorporating pre-characterized $\mathrm{CB}$ and GNPs into a natural rubber matrix at various loadings (Table 1). The speed of the slow roll was $25 \mathrm{~min}^{-1}$. The experiments were repeated for verify ing the statistical significance. The ready compounds in the form of sheets stayed 24 hours prior to their vulcanization.

The optimal vulcanization time was determined by the vulcanization isotherms, taken on an oscillating disc vulcameter MDR 2000 (Alpha Technologies) at $150^{\circ} \mathrm{C}$ according to ISO 3417:2002.

These composites were evaluated for their dielectric (dielectric permittivity, dielectric loss angle tangent) and microwave (reflection coefficient, attenuation coefficient, shielding effectiveness) properties in the $1-12 \mathrm{GHz}$ frequency range.

\subsection{Me asurements}

\subsubsection{Reflection and Attenuation}

Measurements of reflection and attenuation were carried out using the measurement of output (adopted) power $P_{a}$ in the output of a measuring line without losses, where samples of materials may be included. Because of the wide frequency measurement a coaxial line was used. Samples of the materials were shaped into discs with an external diameter $\mathrm{D}=20.6 \mathrm{~mm}$, equal to the outer diameter of the coaxial line and thickness of $\Delta \approx 2 \mathrm{~mm}$. The internal diameter depended on the relative dielectric permittivity of the material.

The sample reflected a part of the incident electro magnetic wave with power $P_{i n}$. The rest of the wave with power $P_{p}$ penetrated the material, so that the attenuation L depended on the coefficient of reflection $|\Gamma|$. Its module was determined by a reflect meter. Thus the attenuation was determined by

$$
L=10 \log \frac{P_{a}}{P_{p}}, d B
$$

where

$$
P_{p}=P_{i n .}\left(1-|\Gamma|^{2}\right)
$$

The following scheme presents the equipment used for testing both parameters (Figure 3)

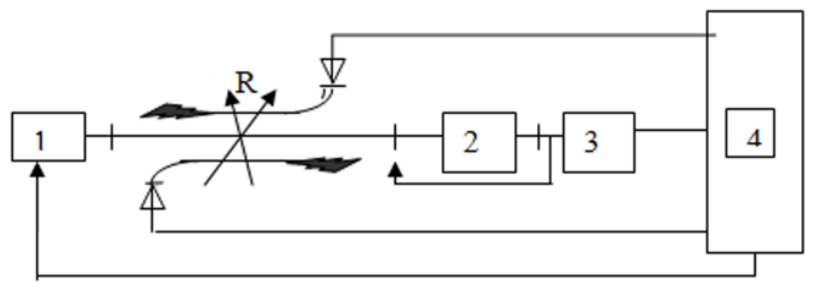

1 - a set of generators for the whole range:HP686A and G4 - 79 to 82;

2 - Coaxial section of the deck E2M Orion, with samples of material;

3 - Power meter HP432A;

4 - Scalar reflectometer HP416A;

$\mathrm{R}$ - Reflectometer

Figure 3. Scheme of the equipment for measuring the microwave properties

\subsubsection{Shielding Effectiveness (S. E.)}

This parameter is defined as the sum of the reflection losses $R, d B$ and attenuation $L, d B$ in the material[31].It can be directly measured or calculated from the measured reflectance and attenuation in the material. In the first case, as measured: incident power on the sample $P_{i n}$, and adopted after the sample $P_{a}$, S.E. is determined by

$$
\text { S.E. }=10 \log \frac{P_{0}}{P_{a}}, d B
$$

In the second, if known reflection and absorption in the material, S.E. is determined, by a definition, as

$$
\text { S.E. }=R, d B+L, d B
$$

where $R, d B$ is the attenuation due to the reflection of power at the interfaces.

In the present work the shielding effectiveness was determined by equation (4).

\subsubsection{Complex Permittivity}

The determination of comp lex permittiv ity was carried out by the resonance method, based on the cavity perturbation technique[32].

Having measured the resonance frequency of the empty cavity resonator $f_{r}$ a measurement of the shift in resonance frequency $f_{\varepsilon}$ was carried out in the presence of the sample material. Then the dielectric constant $\varepsilon_{r}$ was calculated from the shift in resonance frequency, cavity and the sample cross sections $S_{r}$ and $S_{\varepsilon}$, respectively

$$
\varepsilon_{r}=1+\frac{S_{r}}{2 S_{\varepsilon}} \cdot \frac{f_{r}-f_{\varepsilon}}{f_{r}} .
$$

The sample was in the form of a disc with a diameter of 10 $\mathrm{mm}$ and about $2 \mathrm{~mm}$ thick. It was placed at the maximum electric field location of the cavity. Because the thickness of the sample was not equal to the height of the resonator, a dielectric occurred with an equivalent permittivity $\varepsilon_{e}$ at the place of its inclusion. The parameter was determined by (5) and instead $\varepsilon_{r}$ be saved $\varepsilon_{e}$. Then $\varepsilon_{r}$ was determined by 


$$
\varepsilon_{r} \approx \varepsilon_{e}(k+1)-k, \quad(\Delta<<l),
$$

where $k=l / \Delta$ and $l$ is the distance from the disc to the top of the resonator.

\subsubsection{Loss Factor $\tan \delta$}

The loss factor $\tan \delta$ was calculated from the quality factor of the cavity with $Q_{\varepsilon}$ and without sample $Q_{r}$

$$
\tan \delta=\frac{1}{4 \varepsilon_{r}} \frac{S_{r}}{S_{\varepsilon}}\left(\frac{1}{Q_{\varepsilon}}-\frac{1}{Q_{r}}\right) .
$$

The measurement setup used several cavity resonators for the whole range, generators for the whole range, frequency meter and oscilloscope.

The following scheme presents the set used for measuring the dielectric properties (Figure 4):

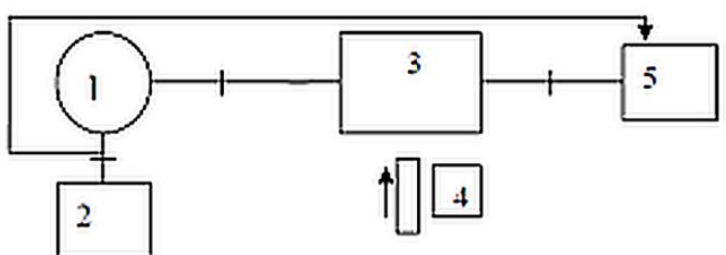

1 - Generators for the whole range: HP686A and G4 - 79 to 82;

2 - Frequency meters: H 532A; FS - 54;

3 - Cavity resonator;

4 - Sample;

5 - Oscilloscope EO 213.

Figure 4. Scheme of the equipment for measuringthe dielectric properties

\section{Results and Discussion}

\subsection{Dielectric Properties}

\subsubsection{Complex Permittivity}

The dependence of the real part of complex dielectric permittivity (the so-called relative dielectric permittivity $\varepsilon_{\mathrm{r}}$ ) on the frequency and amount of the second filler is shown in Figure5.

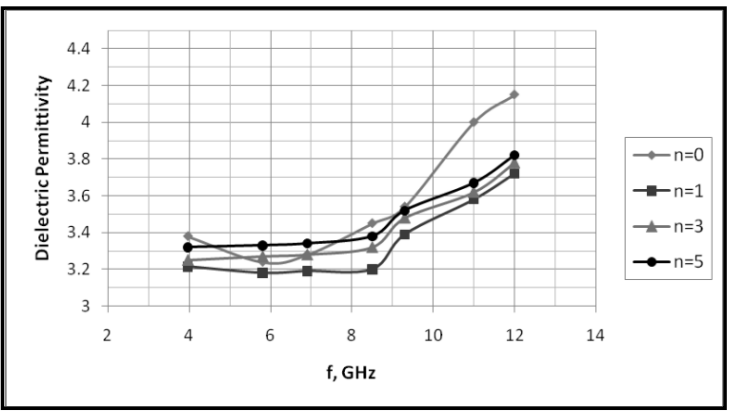

Figure 5. Frequency dependence of relative dielectric permittivity at constant first filler amount (CB-50 phr) and various second filler amount (n-phr of GNP)

It can be seen from the graphs that with the increase of frequency and amount of the second filler (GNP) at constant amount of carbon black ( $50 \mathrm{phr}$ ), the values of relative dielectric permittivity increase. The influence of the frequency and amount of the second filler is significantly pronounced in the range 7-12 GHz and the values of $\varepsilon_{r}$ are between 3.2 and 3.8 .

The polarization mechanis $m$ operating in the gigahertz frequency is purely electronic or orientational with relaxation times smaller than the time period of the applied signals. Interfacial polarization, which is the basic reason for the dispersion in dielectric permittivity at radio frequency regime, has no role to play in microwave frequencies as it does not produce dispersion in $\varepsilon_{\mathrm{r}}$ because of its much smaller relaxation time. But $\varepsilon_{\mathrm{r}}$ was found to increase with the increase of phr of graphene nanoplatelets in the composite as it is evident from Figure 5. This phenomenon of increase in dielectric permittivity with the increase in second filler concentration can be attributed to the enhancement of electrical conductivity of the composites. We consider it was due to graphene, because we have observed similar alterations in the natural rubber based composites with graphene as a filler[33].

\subsubsection{Dielectric Loss}

The dependence of the imaginary part of the complex dielectric permittivity (dielectric losses) on the amount of the second filler and the frequency at constant amount of the first filler is shown in Figure 6.

With the increase of frequency, the dielectric losses decrease, but by increasing the amount of the second filler (GNP) the losses increase. The values of $\tan \delta$ are between 1.7.10 $10^{-3}$ and $13.10^{-3}$.

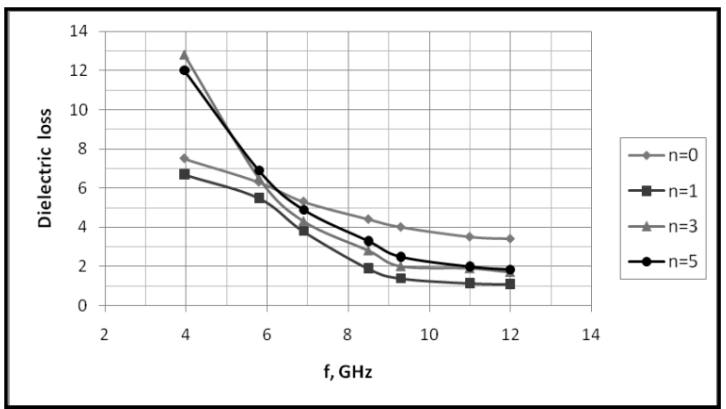

Figure 6. Frequency dependence of dielectric loss at constant first filler amount (50 phr) and various second filler amount (n-phr of GNP)

\subsection{Microwave Properties}

\subsubsection{Coefficient of Reflection}

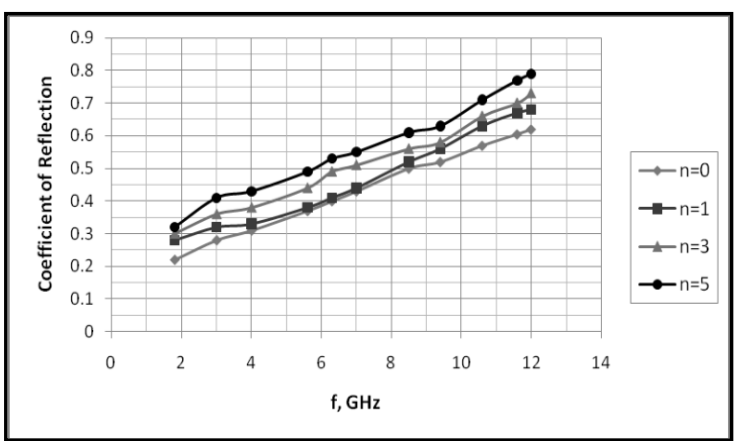

Figure 7. Frequency dependence of coefficient of reflection at constant first filler amount ( $50 \mathrm{phr}$ ) and various second filler amount (n-phrof GNP) 
The dependence of the coefficient of reflection of electromagnetic waves on the frequency and amount of the second filler is shown in Figure 7.

It can be seen from the graphs that with the increase of frequency and amount of the second filler, the reflection coefficient also increases and reaches 0.79 value when $n=5$ and $\mathrm{f}=12 \mathrm{GHz}$. The influence of the amount of GNP is stronger compared to the influence of frequency.

\subsubsection{Coefficient of Attenuation}

The dependence of the coefficients of attenuation on the amount of the second filler and frequency is shown in Figure 8.

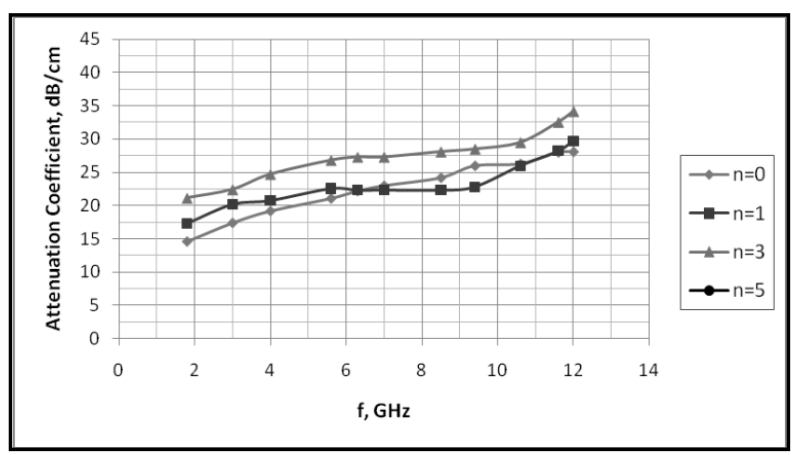

Figure 8. Frequency dependence of coefficient of attenuation at constant first filler amount (50 phr) and various second filler amount (n-phrof GNP)

It is obvious that with the increase of the amount of the second filler and frequency the coefficient of attenuation also increases. When the amount of GNP is higher (e.g. at $5 \mathrm{phr}$, the attenuation coefficient of composites is in the range of $22-39 \mathrm{~dB} / \mathrm{cm})$.

\subsubsection{Shielding Effectiveness}

The dependence of the shielding effectiveness on the amount of the second filler and the frequency is shown in Figure 9. The decrease in the values of this characteristic depending on frequency is quite obvious since the growth of the coefficients of attenuation is apparently not high enough to make up for the increase in reflection coefficients. These two coefficients according to equation (4) are crucial to the formation of the value of the shielding effectiveness.

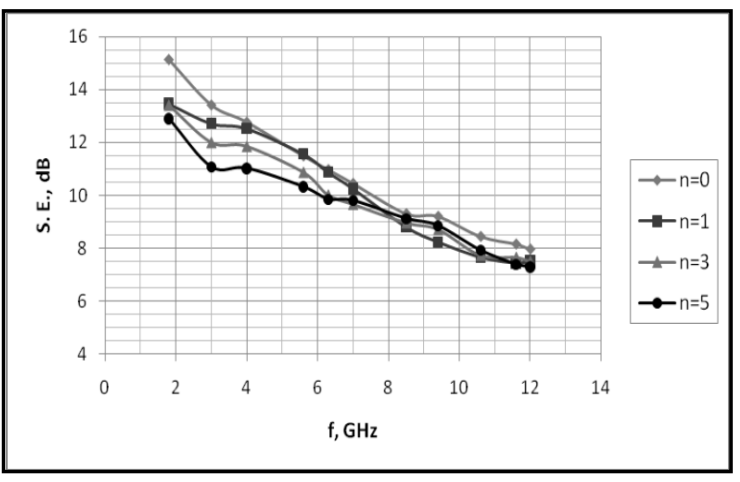

Figure 9. Frequency dependence of electromagnetic interference shielding effectiveness at constant first filler amount (50 phr) and various second filler amount (n-phr of GNP)
It was found out that the percolation threshold for composites filled with the investigated fillers, but individually and not in combination, is at 12 and $15 \mathrm{phr}$ respectively for GNP and CB[34]. It is possible because GNP and CB have different geometry (the values of aspect ratio for $\mathrm{CB}$ is 1 and for GNP - from 120 to 200). According to Balberg[34] the average interparticle distance can be different in the carbon nanofillers with different aspect ratios. The more spherical-like structure, the larger the interparticle distance will be formed. For the elongated particles, their composites have a very narrow distribution of the distances due to the entangled particles structures [35] and the volume resistivity of the composites decreases monotonically with increasing the aspect ratio. The interparticle distance, composites microstructure and the volume resistivity have a strong influence on the microwave properties [36]. Presumably the increase of the dielectric permittivity of the composites, containing GNP and carbon black toward high frequencies is induced by the dielectric relaxation which suggests that the established percolation network structure is not stable and easily affected by the external frequency disturbances.

Our investigations present that adding small a mounts (1-5 phr) of GNP to a standard constant amount of active furnace carbon black can be used as a way to modify and to improve the dielectric (dielectric permittivity, dielectric loss) and microwave properties (coefficient of reflection, coefficient of attenuation, electromagnetic interference shielding effectiveness) of natural rubber based composites in the high frequency range $1-12 \mathrm{GHz}$.

\section{Conclusions}

NR based nanocomposites containing a constant amount of standard furnace carbon black and GNP in concentrations fro $\mathrm{m} 1$ to $5 \mathrm{phr}$ have been prepared. Their dielectric (dielectric permittivity, dielectric loss) and microwave properties (coefficients of absorption and reflection of the electromagnetic waves and electromagnetic interference shielding effectiveness) have been investigated in the $1-12 \mathrm{GHz}$ frequency range. It was found effect of modifying the properties of the composites. Adding small amounts of GNP to a constant amount of carbon black can be used as a way to control and primarily to improve the dielectric and microwave properties of composites based on natural rubber in the high frequency range 1-12 GHz. The results achieved allow us to recommend graphene as second filler for natural rubber based composites to afford specific absorbing properties.

\section{ACKNOWLEDGEMENTS}

The present research is a result of an international collaboration program between University of Tabuk, Tabuk, Kingdom of Saudi Arabia and the University of Chemical Technology and Metallurgy, Sofia, Bulgaria. The authors gratefully acknowledge the financial support from the Uni- 
versity of Tabuk.

\section{REFERENCES}

[1] S. Thomas, R. Stephen, "Rubber Nanocomposites Preparation, Properties and Applications", Wiley, Singapore, 2010

[2] P. M. Ajayan, "Single-walled carbon nanotube-polymer composites: Strength and weakness", Advanced Materials, Vol. 12, No.10, pp. 750-753, 2000

[3] E. T. Thostenson, "Advances in the science and technology of carbon nanotubes and their composites: a review", Composites Science and Technology, Vol. 61, No. 13, pp. 1899-1912, 2001

[4] A. Krueger, "Carbon Materials and Nanotechnology", Wiley-VCH, Verlag GmbH\& Co. KGaA, 2010

[5] K. S. Novoselov, "Electric Field Effect in Atomically Thin Carbon Films", Science, Vol. 306, No 5696, pp. 666-669, 2004

[6] H. J. Salavagione, G. Martínez , G. Ellis, "Graphene-Based Polymer Nanocomposites" in Sergey Mikhailov, Ed., Physics and Applications of Graphene - Experiments, InTech, pp. 169-192, 2011

[7] M. J. Allen, V. C. Tung and R. B. Kaner, "Honey comb carbon: A Review of Graphene", Chem. Rev., Vol. 110, No. 1, pp. 132-145, 2010

[8] Y. Zhu, S. Murali, W. Cai, X. Li, J. W. Suk, J. R. Potts, R. S. Ruoff, "Graphene and Graphene Oxide: Synthesis, Properties, and Applications", Adv. Mater., Vol. 22, No. 35, pp. 1-19, 2010

[9] O. C. Compton, S. T. Nguyen, "Graphene Oxide, Highly Reduced Graphene Oxide. and Graphene: Versatile Building Blocks for Carbon-Based Materials", Small, Vol. 6, No. 6, pp. 711-723, 2010

[10] P. Mukhopadhyay, R. K. Gupta, "Trend and Frontiers in Graphene-Based Polymer Nanocomposites", Plastics Engineering, pp.32-42, 2011, www.4spe.org

[11] R. K. Prud'Homme, B. Ozbas, I. Aksay, R. Register, D. Adamson - U.S. Patent 7,745,528 June 29, 2010

[12] S. Pacchini, T. Idda, D. Dubuc, E. Flahaut, K. Grenier, "Carbon nanotube-based polymer composites for microwave applications", Microwave Svmbosium Digest. IEEE MTT-S International, Conference Publications, pp. 101 - 104, 2008

[13] D. Micheli, R. Pastore, C. Apollo, M. Marchetti, G. Gradoni, F. Moglie, V.M. Primiani, "Carbon based nanomaterial composites in RAM and microwave shielding applications", Nanotechnology, IEEE-NANO 2009. 9th IEEE Conference on, Conference Publications, pp.226-235, 2009

[14] A. Saib, L. Bednarz, R. Daussin. C. Bailly, Xudong Lou, J.-M. Thomassin, C. Pagnoulle, C. Detrembleur, R. Jerome, I. Huynen, "Carbon nanotube composites for broadband microwave absorbing materials", Microwave Theory and Techniques, IEEE Transactions on, Vol. 54, No. 6, pp. 2745 $-2754,2006$

[15] Y. Zhai, Y. Zhang, W. Ren, "Electromagnetic characteristic and microwave absorbing performance of different carbon-based hydrogenated acrylonitrile - butadiene rubber composites", Materials Chemistry and Physics, Vol. 133, No. 1, pp. 176-181, 2012

[16] Z. Liu, G. Bai, Yi Huang, F. Li, Y. Ma, T. Guo, X. He, X. Lin, H. Gao, and Y. Chen, "Microwave Absorption of Single-Walled Carbon Nanotubes/Soluble Cross-Linked Polyurethane Composites", J. Phys. Chem. C, Vol. 111, 13696-13700, 2007

[17] Y. Xu, D. Zhang, J. Cai, L. Yuan and W. Zhang, "Effects of Multi-walled Carbon Nanotubes on the Electromagnetic Absorbing Characteristics of Composites Filled with Carbonyl Iron Particles", J. Mater. Sci. Technol., Vol. 28, No. 1, pp. 34-40, 2012

[18] H. Li , J. Wang, Y. Huang, X. Yan, J. Qi, J. Liu, Y. Zhang, "Microwave absorption properties of carbon nanotubes and tetrapod-shaped $\mathrm{ZnO}$ nanostructures composites", Materials Science and Engineering B Vol.175, pp. 81-85, 2010

[19] L. Liu, L. B. Kong, W. Y. Yin, Y. Chen and S. Matitsine, "Microwave Dielectric Properties of Carbon Nanotube Composites", in J.M.Marulanda, Ed., Carbon Nanotubes, InTech, pp. 93-108, 2010

[20] H.Y. Zhang, G.X. Zeng, Y. Ge, T.L. Chen and L.C.Hu: "Electromagnetic characteristic and microwave absorption properties of carbon nanotubes/epoxy composites in the frequency range from 2 to $6 \mathrm{GHz}$ " J. Appl. Phy s., Vol. 105, No. 5, 054314, 2009

[21] Z.F. Liu, G. Bai, Y. Huang, Y.F. Ma, F. Du, F.F. Li, T.Y. Guo and Y.S. Chen, "Reflection and absorption contributions to the electromagnetic interference shielding of single-walled carbon nanotube/polyurethane composites", Carbon, Vol. 45, No. 4, pp. 821-827, 2007

[22] S. Gunasekaran, R.K. Natarajan, A. Kala and R. Jagannathan, "Dielectric studies of some rubber materials at microwave frequencies", Indian Journal of Pure\&Applied Phy sics, Vol. 46, pp. 733-737, 2008

[23] A.M. Shanmugharaj, J.H. Bae, Kwang Yong Lee, Woo Hyun Noh, Se Hyoung Lee, Sung Hun Ryu, "Physical and chemical characteristics of multiwalled carbon nanotubes functionalized with aminosilane and its influence on the properties of natural rubber composites", Composites Science and Technology, Vol. 67, pp. 1813-1822, 2007

[24] G. Sui, W. Zhong, X. Yang, and S. Zhao, "Processing and material characteristics of a carbon-nanotube-reinforced natural rubber", Macromolecular Materials and Engineering, Vol. 292, No.9, pp.1020-1026, 2007

[25] M. Kolodziej, L. Bokobza, and J.L. Bruneel, "Investigations on natural rubber filled with multiwall carbon nanotubes", Composite Interfaces, Vol.14, No.3, pp. 215-228, 2007

[26] J. Liang, Y. Huang, Y. Ma, Z. Liu, J. Cai, Ch. Zhang, H. Gao, Y. Chen, "Electromagnetic interference shielding of graphene/ep oxy composites", Carbon, Vol. 47, No. 3, pp. 922-925, 2009

[27] I. M. De Rosa, F. Sarasini, M. S. Sarto, A. Tamburrano, "EMC impact of advanced carbon fiber/carbon nanotube reinforced composites for next generation aerospace applications", IEEE Trans. on EMC, Special Issue "EMC in Aerospace", Vol. 50, No. 3, pp. 556-563, 2008 
[28] I. M. De Rosa, R. Mancinelli, F. Sarasini, M. S. Sarto, A. Tamburrano, "Electromagnetic Design and Realization of Innovative Fiber-Reinforced Broad-B and Absorbing Screens", IEEE Trans. on Electromagnetic Compatibility, Vol. 51, No. 3, pp. $700-707,2009$

[29] I. M. De Rosa, A. Dinescu, F. Sarasini, M. S. Sarto, A. Tamburrano, "Effect of short carbon fibers and MWCNTs on microwave absorbing properties of polyester composites containing nickel-coated carbon fibers", Composites Science and Technology, Vol. 70, No. 1, pp. 102-109, 2010

[30] G. De Bellis, I. M. De Rosa, A. Dinescu, M. S. Sarto, A. Tamburrano, "Electromagnetic Absorbing Nanocomposites Including Carbon Fibers, Nanotubes and Graphene Nanoplatelets", Proceedings of the 2010 IEEE International Sy mposium on Electromagnetic Compatibility, pp. 202-207, 2010

[31] C.R. Paul, "Introduction to Electromagnetic Comp atibility", John Wiley \& Sons, New York, 1992

[32] B. Meng, J. Booske, and R. Cooper, "Extended cavity perturbation technique to determine the complex permittivity of the dielectric materials", IEEE T. Microw. Theory Tech., Vol. 43, pp. 2633-2636, 1995

[33] O. A. Al-Hartomy, A. Al-Ghamdi, N. Dishovsky, R. Shtarkova, V. Iliev, I. Mutlay, F. El-Tantawy, "Dielectric and Microwave Properties of Natural Rubber Based Nanocomposites Containing Graphene", Materials Sciences and Applications, Vol. 3, 2012, Published Online July 2012 (http://www.SciRP.org/journal/msa)

[34] Y. Li, J. Zhu, S. Wei, J. Ryu, Q. Wang, L. Sun and Z. Guo, "Poly (propylene) Nanocomposites Containing Various Carbon Nanostructures", Macromol. Chem.Phys., Vol. 212, No. 22, pp. 2429-2438, 2011

[35] I. Balberg, "A comprehensive picture of the electrical phenomena in carbon black-polymer composites", Carbon, Vol. 40, No. 2, pp.139-143, 2002

[36] N. Dishovsky, "Rubber Based Composites with active Behaviour to Microwaves" (Review),Journal of the University of Chemical Technology and Metallurgy, Vol. 44, No. 2, pp.115-122, 2009 